\title{
Numerical Simulation of the Instability of a Non- Isothermal Liquid Film in Film Devices
}

\author{
L.A. Prokudina ${ }^{1}$, and M.P. Vikhirev ${ }^{1, *}$ \\ ${ }^{1}$ South Ural State University, 454080 Chelyabinsk, Russian Federation
}

\begin{abstract}
This work presents results of numerical simulation of the instability of vertical liquid film flow at moderate Reynolds numbers in the framework of partial differential equation of the state of the free surface of the liquid film. Equation coefficients include different physicochemical factors such as surface tension parameter and Marangoni parameter. Python scripts are used for computation of wave characteristics, instability regions of liquid films and liquid films free surface state. Computational experiments were carried out for the vertical water and alcohol films. Results could be used in the design or modernization of existing equipment and in the design of technological processes in liquid films.
\end{abstract}

\section{Introduction}

Liquid film is an effective tool of interfacial heat and mass transfer due to large contact surface area and low liquid flow rate [1-3], so it is widely used in numerous industry fields (chemical, petrochemical, energy, food, etc.). The efficiency of film devices based on the flow of thin liquid films is due to the large contact surface, low thermal resistance and low power consumption. The flow of liquid films is unstable, which leads to the appearance of nonlinear surface waves. Falling film devices are used for cooling or heating of low viscosity liquids: alcohols, dyes, organic solvents, antibiotics, vitamins, etc.

Influence of different physicochemical factors on liquid film flow regimes should be considered when designing falling film devices [4-6]. Such factors include liquid surface tension, gradients of temperature and concentration of substances [7, 8].

The aim of this work is numerical study of wave characteristics of the water and alcohol films flow considering moderate Reynolds numbers.

\section{Mathematical model of liquid film free surface state}

We consider the flow of a thin viscous liquid layer with a thickness $\delta$ on a vertical solid heated surface. Denote deviation of the liquid film free surface from the undisturbed state by $\psi(x, t)$. Taking into account, that surface tension $\sigma$ is a function of liquid temperature $T$ and liquid film surface chemical composition, we introduce dimensionless parameter

\footnotetext{
${ }^{*}$ Corresponding author: vikhirevmp@susu.ru
} 
$M=\frac{\partial \sigma}{\partial T}\left(\frac{\partial \sigma}{\partial y}\right)_{y=\delta} \frac{1}{u_{0}^{2} \rho}$, called Marangoni number, where $u_{0}$ - fluid flow average velocity, $\rho$ - fluid density.

Liquid film free surface state described by following partial differential equation [7]:

$$
\begin{aligned}
& b_{1} \frac{\partial \psi}{\partial x}+b_{2} \frac{\partial^{2} \psi}{\partial x^{2}}+b_{4} \frac{\partial^{4} \psi}{\partial x^{4}}+b_{5} \psi \frac{\partial \psi}{\partial x}+b_{6} \psi \frac{\partial^{2} \psi}{\partial x^{2}}+b_{7}\left(\frac{\partial \psi}{\partial x}\right)^{2}+b_{9} \psi\left(\frac{\partial \psi}{\partial x}\right)^{2}+ \\
& +\left(b_{10} \frac{\partial}{\partial x}-1\right) \frac{\partial \psi}{\partial t}=0
\end{aligned}
$$

where $\quad b_{1}=-R e F_{x}, \quad b_{2}=-\frac{1}{2} \operatorname{ReM}+\frac{3}{40} \operatorname{Re}^{3} F_{x}^{2}, \quad b_{4}=-\frac{1}{3} \operatorname{Re\sigma }, \quad b_{5}=-2 R e F_{x}$, $b_{6}=b_{7}=-\operatorname{ReM}+\frac{9}{20} R e^{3} F_{x}^{2}, \quad b_{9}=-\operatorname{ReM}+\frac{9}{4} \operatorname{Re}^{3} F_{x}^{2}, \quad b_{10}=\frac{5}{24} R e^{2} F_{x}, R e-$ Reynolds number, $F_{x}$ - Froude number.

In heat and mass transfer processes, including liquid film evaporation process, high temperature gradients could cause destruction of liquid film and formation of "dry spot" on film device surface. Marangoni number critical values, at which destruction of film is possible, are given by the following formula:

$$
M_{c}=\frac{3}{20} \operatorname{Re}^{2} F_{x}^{2}-\frac{2}{k^{2} R e}\left(a_{0}-\frac{a_{1}}{a_{2}}\right),
$$

where $a_{0}=b_{4} k^{4}, a_{1}=-b_{1} k, a_{2}=-b_{10} k$.

\section{Computational experiments}

We consider the linear part of equation (1). Using the solution type $\psi=A \exp (i k x-\omega t)$ we obtain the following dispersion equation:

$$
\omega\left(b_{10} k+i\right)+b_{4} k^{4}-b_{2} k^{2}+b_{1} i k=0,
$$

where $\omega=\omega_{r}+i \omega_{i}\left(\omega_{r}-\right.$ frequency, $\omega_{i}-$ increment $)$.

Phase velocity values could be calculated by the following formula:

$$
c_{r}=\frac{\omega_{r}}{k}
$$

Computational experiments were carried out for the vertical water film at Reynolds numbers $1 \leq R e \leq 15$ and wave numbers $0 \leq k \leq 0.5$. Free flow of water and alcohol liquid films was studied. Python script, calculating frequency, increment, phase velocity values and Marangoni number critical values, was developed.

Flow of vertical liquid film is unstable at positive increment values. The criterion of instability of the flow of liquid films is $\omega_{i}>0$. 
Flow of alcohol film is more unstable. The instability region of the alcohol film increases almost twice as much as the instability region of the water film, as shown in the table 1 .

Table 1. Instability regions.

\begin{tabular}{|c|c|c|c|l|c|}
\hline \multirow{2}{*}{$R e$} & \multicolumn{3}{|c|}{$k$} & \multirow{2}{*}{$R e$} & \multicolumn{2}{|c|}{$k$} \\
\cline { 2 - 3 } \cline { 5 - 6 } & Water & Alcohol & & Water & Alcohol \\
\hline 1 & {$[0 ; 0.0272]$} & {$[0 ; 0.0471]$} & 9 & {$[0 ; 0.1694]$} & {$[0 ; 0.2934]$} \\
\hline 2 & {$[0 ; 0.0484]$} & {$[0 ; 0.0838]$} & 10 & {$[0 ; 0.185]$} & {$[0 ; 0.3203]$} \\
\hline 3 & {$[0 ; 0.0679]$} & {$[0 ; 0.1175]$} & 11 & {$[0 ; 0.2002]$} & {$[0 ; 0.3468]$} \\
\hline 4 & {$[0 ; 0.0862]$} & {$[0 ; 0.1493]$} & 12 & {$[0 ; 0.2153]$} & {$[0 ; 0.3729]$} \\
\hline 5 & {$[0 ; 0.1038]$} & {$[0 ; 0.1798]$} & 13 & {$[0 ; 0.2301]$} & {$[0 ; 0.3986]$} \\
\hline 6 & {$[0 ; 0.1209]$} & {$[0 ; 0.2093]$} & 14 & {$[0 ; 0.2448]$} & {$[0 ; 0.424]$} \\
\hline 7 & {$[0 ; 0.1374]$} & {$[0 ; 0.238]$} & 15 & {$[0 ; 0.2593]$} & {$[0 ; 0.4491]$} \\
\hline 8 & {$[0 ; 0.1536]$} & {$[0 ; 0.266]$} & & & \\
\hline
\end{tabular}

Calculated increment values are presented in figures 1 and 2.

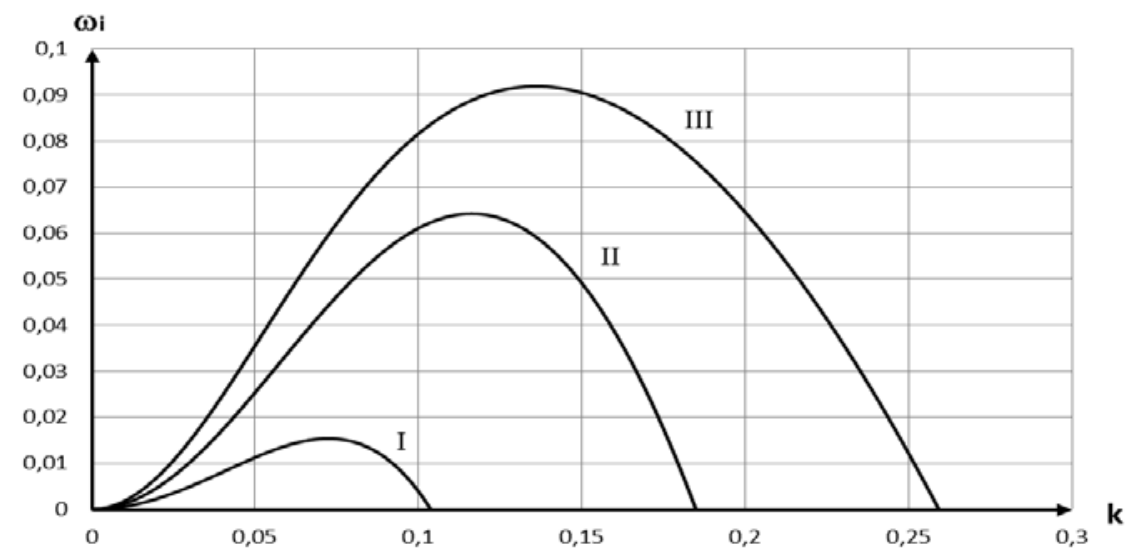

Fig. 1. Increment values of water film flow: $\mathrm{I}-R e=5, \mathrm{II}-R e=10, \mathrm{III}-R e=15$.

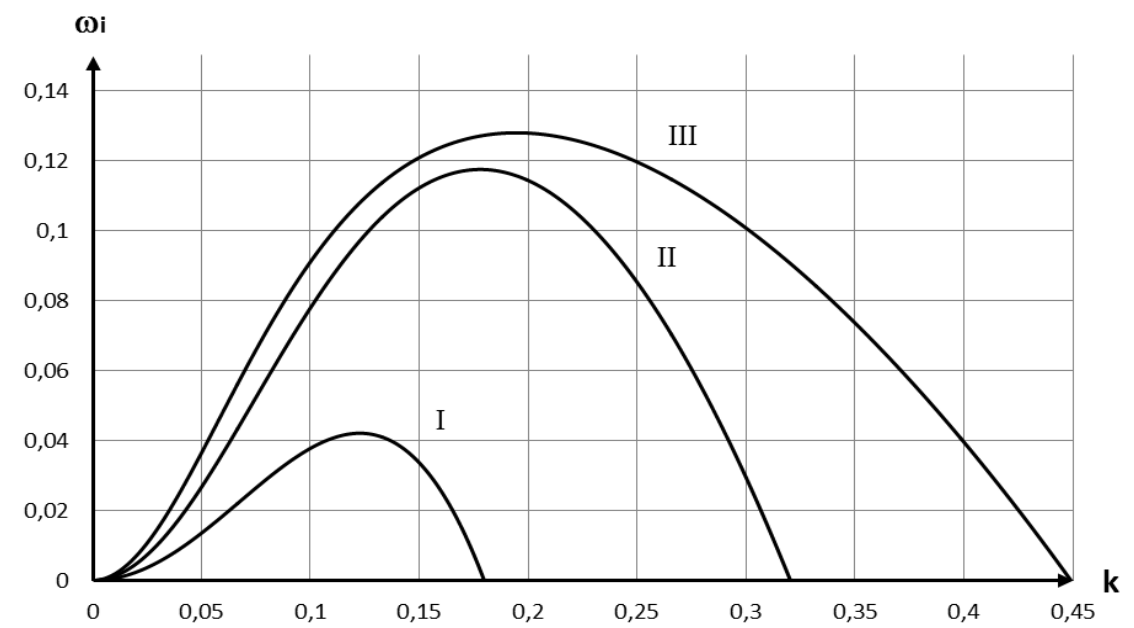

Fig. 2. Increment values of alcohol film flow: $\mathrm{I}-R e=5$, II $-R e=10$, III $-R e=15$. 
In the areas of instability of both the liquid film of water and alcohol, the increment value reaches its maximum value (Fig. 1, 2). Such modes are called optimal, they are recorded in experimental studies of liquid films.

Figures 3 and 4 present calculated phase velocity values.

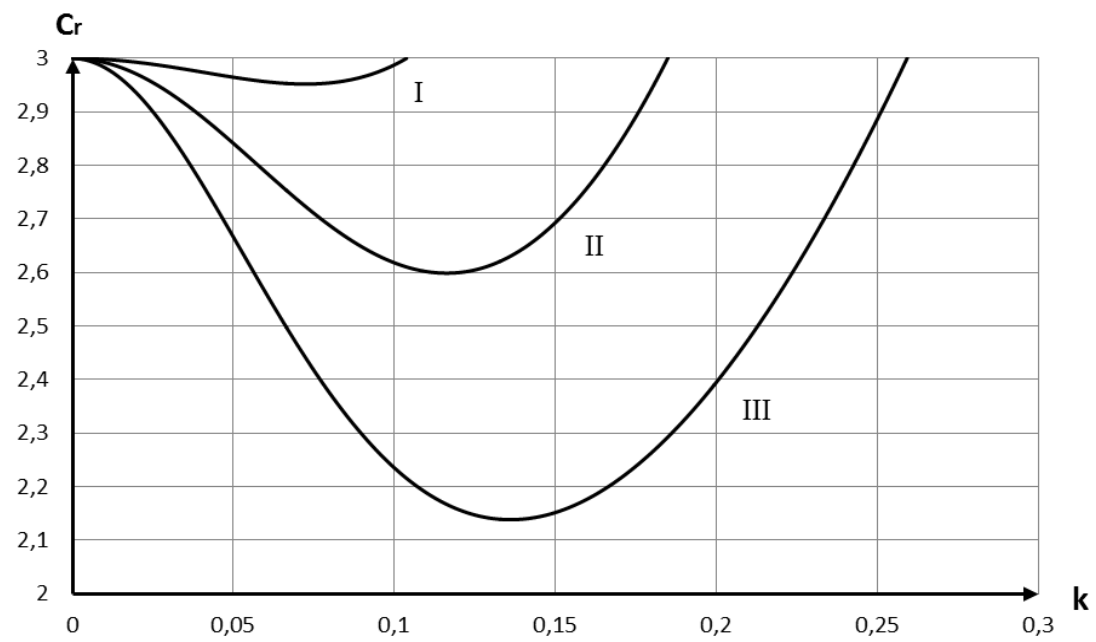

Fig. 3. Phase velocity values of water film flow: $\mathrm{I}-R e=5$, II $-R e=10$, III $-R e=15$.

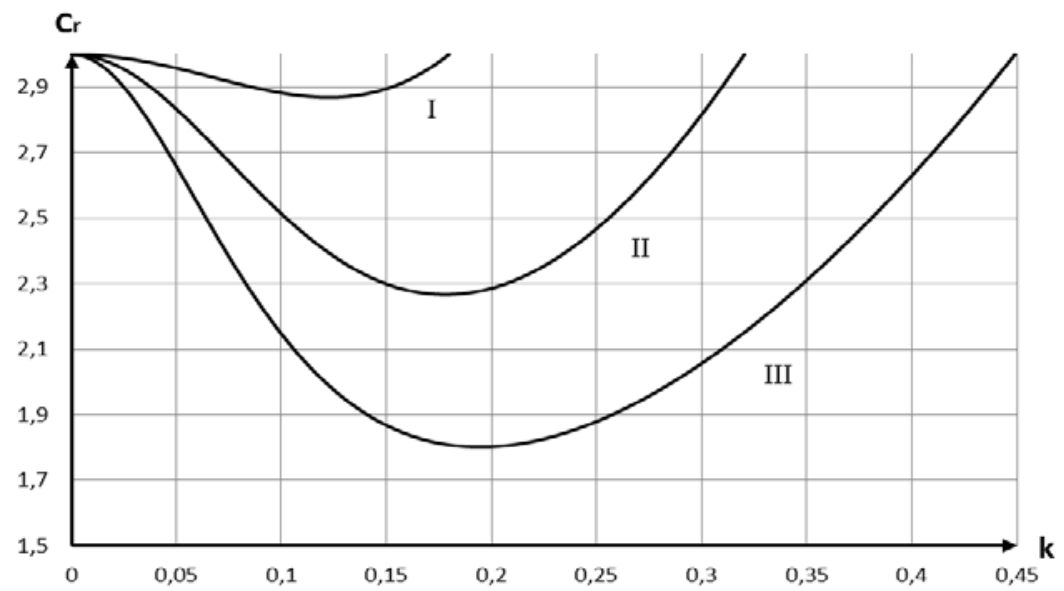

Fig. 4. Phase velocity values of alcohol film flow: I $-R e=5$, II $-R e=10$, III $-R e=15$.

It can be noted that the minimum value of the phase velocity corresponds to the maximum value of the increment. Table 2 shows maximum values of increment at different Reynolds numbers and corresponding values of phase velocity. We could note increase of increment maximum values and decrease of corresponding phase velocity values for alcohol film flow. For Reynolds numbers less than eight, the phase velocity values are sufficiently high, on the order of three. For Reynolds numbers greater than eight, there is a noticeable decrease in the phase velocity, especially for alcohol films. 
Table 2. Increment and phase velocity.

\begin{tabular}{|l|l|l|l|l|l|l|}
\hline \multirow{2}{*}{$R e$} & \multicolumn{3}{|c|}{ Water } & \multicolumn{3}{c|}{ Alcohol } \\
\cline { 2 - 7 } & \multicolumn{1}{|c|}{$k$} & $\omega_{\text {imax }}$ & \multicolumn{1}{|c|}{$c_{r}$} & \multicolumn{1}{c|}{$k$} & \multicolumn{1}{c|}{$\omega_{i \max }$} & \multicolumn{1}{c|}{$c_{r}$} \\
\hline 1 & 0.0192 & 0.000221 & 2.999862 & 0.0332 & 0.000663 & 2.999586 \\
\hline 2 & 0.0342 & 0.001401 & 2.998249 & 0.0591 & 0.004187 & 2.994767 \\
\hline 3 & 0.0478 & 0.004104 & 2.992305 & 0.0825 & 0.012119 & 2.977276 \\
\hline 4 & 0.0606 & 0.008709 & 2.978227 & 0.1038 & 0.025017 & 2.937458 \\
\hline 5 & 0.0725 & 0.015357 & 2.952009 & 0.1227 & 0.042057 & 2.868573 \\
\hline 6 & 0.0834 & 0.023877 & 2.910463 & 0.1388 & 0.061117 & 2.770812 \\
\hline 7 & 0.0933 & 0.033763 & 2.852287 & 0.1522 & 0.079644 & 2.651556 \\
\hline 8 & 0.1021 & 0.044279 & 2.778603 & 0.1629 & 0.095681 & 2.521594 \\
\hline 9 & 0.1098 & 0.05464 & 2.692653 & 0.1714 & 0.1083 & 2.390813 \\
\hline 10 & 0.1163 & 0.064189 & 2.598821 & 0.178 & 0.11743 & 2.26606 \\
\hline 11 & 0.1218 & 0.072502 & 2.501549 & 0.1831 & 0.123479 & 2.151082 \\
\hline 12 & 0.1264 & 0.079388 & 2.404587 & 0.1871 & 0.127021 & 2.047341 \\
\hline 13 & 0.1303 & 0.084838 & 2.31069 & 0.1902 & 0.128628 & 1.954901 \\
\hline 14 & 0.1335 & 0.088956 & 2.221635 & 0.1927 & 0.12879 & 1.873086 \\
\hline 15 & 0.1361 & 0.091903 & 2.138409 & 0.1946 & 0.127905 & 1.800894 \\
\hline
\end{tabular}

High temperature gradients contribute to an increase in the increment and a drop in the phase velocity, which can lead to the destruction of liquid films and affect the ongoing technological process in the films, up to the creation of an emergency situation in the operation of film equipment. Marangoni number critical values, calculated at maximum increment values, are presented in table 3.

Table 3. Marangoni number critical value.

\begin{tabular}{|c|c|c|c|c|c|c|c|c|c|}
\hline \multirow{2}{*}{$\operatorname{Re}$} & \multicolumn{2}{|c|}{ Water } & \multicolumn{2}{|c|}{ Alcohol } & \multirow{2}{*}{$\operatorname{Re}$} & \multicolumn{2}{|c|}{ Water } & \multicolumn{2}{|c|}{ Alcohol } \\
\hline & $k$ & $M_{c}$ & $k$ & $M_{c}$ & & $k$ & $M_{c}$ & $k$ & $M_{c}$ \\
\hline 1 & 0.0192 & 26044.2 & 0.0332 & 8712.1 & 9 & 0.1098 & 12.2 & 0.1714 & 6.2 \\
\hline 2 & 0.0342 & 2054.5 & 0.0591 & 689.7 & 10 & 0.1163 & 9.4 & 0.178 & 5.1 \\
\hline 3 & 0.0478 & 469.4 & 0.0825 & 159.3 & 11 & 0.1218 & 7.6 & 0.1831 & 4.4 \\
\hline 4 & 0.0606 & 165.9 & 0.1038 & 58.2 & 12 & 0.1264 & 6.4 & 0.1871 & 3.9 \\
\hline 5 & 0.0725 & 75.6 & 0.1227 & 28 & 13 & 0.1303 & 5.5 & 0.1902 & 3.5 \\
\hline 6 & 0.0834 & 40.8 & 0.1388 & 16.2 & 14 & 0.1335 & 4.8 & 0.1927 & 3.2 \\
\hline 7 & 0.0933 & 25 & 0.1522 & 10.8 & 15 & 0.1361 & 4.3 & 0.1946 & 2.9 \\
\hline 8 & 0.1021 & 16.8 & 0.1629 & 7.9 & & & & & \\
\hline
\end{tabular}

\section{Waves on the free surface of the liquid film}

Liquid film free surface state equation can be written in the following form [8]:

$$
\frac{\partial \psi}{\partial t}=b_{1} \frac{\partial \psi}{\partial x}+b_{2} \frac{\partial^{2} \psi}{\partial x^{2}}+b_{4} \frac{\partial^{4} \psi}{\partial x^{4}}+b_{5} \psi \frac{\partial \psi}{\partial x}+b_{6} \psi \frac{\partial^{2} \psi}{\partial x^{2}}+b_{7}\left(\frac{\partial \psi}{\partial x}\right)^{2}+b_{9} \psi\left(\frac{\partial \psi}{\partial x}\right)^{2}
$$

Following algorithm for calculating liquid film free surface state at different moments of time was implemented on Python:

1) Calculate values of $b_{1}, b_{2}, b_{4}, b_{5}, b_{6}, b_{7}$ and $b_{9}$ coefficients.

2) Partition the spatial and time domains with grid points $x_{i}=i \Delta x$ and $t_{j}=j \Delta t$; $i=0, . ., L_{x}, j=0, . ., L_{t}$. 
3) Interpolate $\psi(x)$ function at moment of time $t_{j}$ using Lagrange interpolating polynomial. Use obtained polymial to calculate partial derivatives $\frac{\partial \psi}{\partial x}, \frac{\partial^{2} \psi}{\partial x^{2}}, \frac{\partial^{3} \psi}{\partial x^{3}}$ and $\frac{\partial^{4} \psi}{\partial x^{4}}$ values for each point $x_{i}$.

4) Calculate $\psi(x)$ function values at moment of time $t_{j}+\Delta t$ by formula (5) using the following substitution: $\left.\frac{\partial \psi}{\partial t}\right|_{x=x_{i}, t=t_{j}}=\frac{\psi\left(x_{i}, t_{j}+\Delta t\right)-\psi\left(x_{i}, t_{j}\right)}{\Delta t}$.

5) Repeat steps 3 and 4 for each $j=0, . ., L_{t}$.

Computational experiments were carried out for investigation of water and alcohol liquid films free surface state at $R e=10$. Following parameters of experiment were used: $\psi(x, 0)=0.001 \sin \left(\frac{2 \pi x}{80}\right), \Delta x=5, \Delta t=0.01, L_{x}=17, L_{t}=501$. Figures 5 and 6 show results of calculation of free surface state at moments of time $t=0, t=2$ and $t=5$.

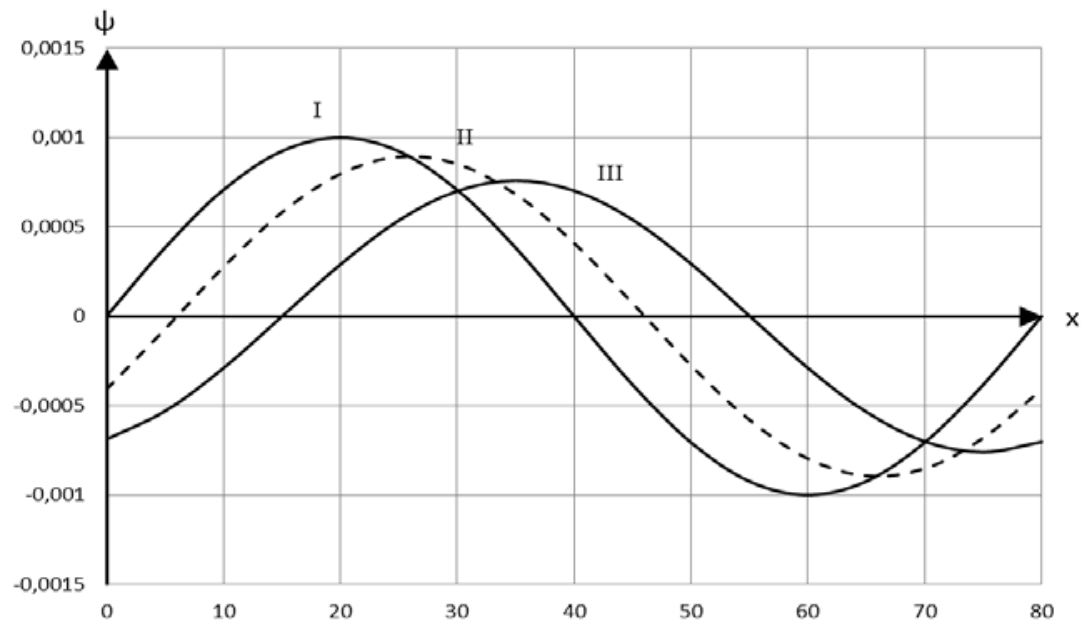

Fig. 5. Water liquid film free state: $\mathrm{I}-t=0, \mathrm{II}-t=2, \mathrm{III}-t=5$.

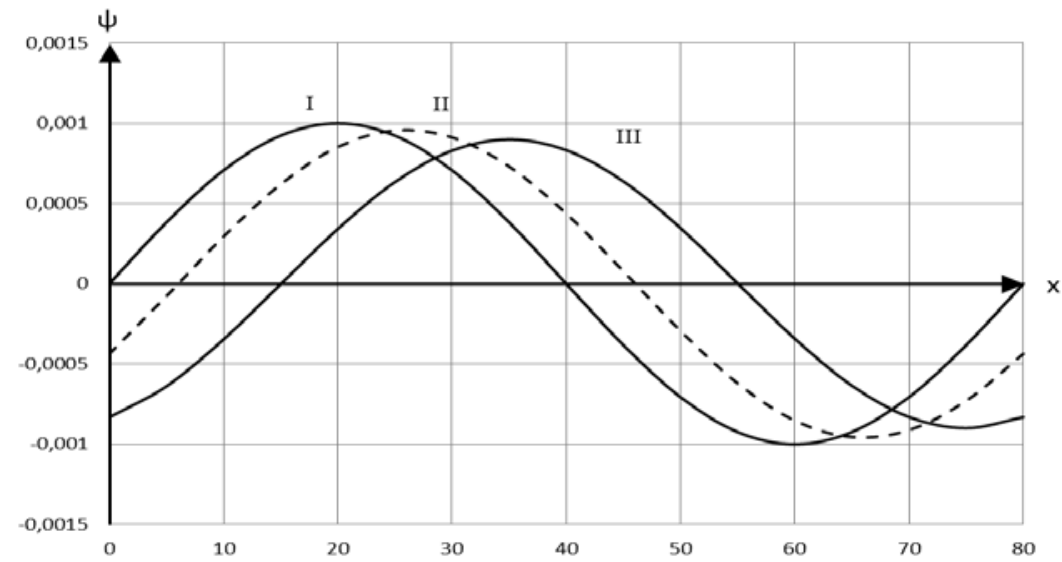

Fig. 6. Alcohol liquid film free state: $\mathrm{I}-t=0, \mathrm{II}-t=2$, III $-t=5$. 
According to the results of computational experiments, it can be noted that waves with an amplitude exceeding the amplitude of waves on the water film are formed on the surface of the alcohol film over the entire studied range of Reynolds numbers.

\section{Conclusion}

1. Mathematical model of free surface state of a viscous fluid thin layer at moderate Reynolds numbers is presented. For the presented mathematical model, computational algorithms and programs for calculating the wave characteristics of liquid films on Python are developed.

2. Numerical study of vertical water and alcohol films flow regimes are carried out. Wave characteristics (frequency, increment, phase velocity) of liquid film flow are calculated. Optimal flow regimes of the liquid film characterized by the maximum increment and the minimum phase velocity are revealed.

3. Marangoni number critical values and instability regions of liquid film flow are calculated. It is possible to note the most destructive effect of temperature gradients on liquid films of alcohol in comparison with liquid films of water. Liquid films of water and alcohol for Reynolds numbers less than four can withstand higher temperature gradients (Table 3).

4. Comparison of water and alcohol film flow shows that alcohol film flow is more unstable: it is characterized by expansion of instability region width and decrease of Marangoni number critical values. Liquid films with low surface tension, which include alcohol films, are more unstable compared to liquid water films.

5. In accordance with the developed computational algorithm, the results of computer simulation of the wave surface of liquid films are presented. The wave amplitudes are calculated. Alcohol films tend to have higher amplitudes. The most developed wave surfaces of liquid films contribute to the intensification of processes in liquid films.

\section{References}

1. S.V. Alekseenko, V.E. Nakoryakov, B.G. Pokusaev, Wave flow of liquid films. Nauka, Novosibirsk (1992)

2. E.G. Vorontsov, Yu.M. Tananayko, Heat and mass transfer in liquid films. Tehnika, Kiev (1972)

3. L.P. Kholpanov, V.Ya. Shkadov, Hydrodynamics and heat and mass transfer with interface. Nauka, Moscow (1990)

4. O.A. Burmistrova, Stability of a vertical liquid film with consideration of the marangoni effect and heat exchange with the environment. Journal of Applied Mechanics and Technical Physics 55, 386-393 (2014)

5. V. Subramaniam, S. Garimella, Numerical study of heat and mass transfer in lithium bromide-water falling films and droplets. International Journal of Refrigeration 40, 211-226 (2014)

6. L.A. Prokudina, Numerical simulation of effect of temperature gradients on flow of liquid film in heat and mass transfer devices. Global Smart Industry Conference (2018)

7. L.A. Prokudina, Influence of surface tension inhomogeneity on the wave flow of a liquid film. Journal of Engineering Physics and Thermophysics 87(1), 165-173 (2014)

8. L.A. Prokudina, Modelling the influence of temperature gradients on the state of a free liquid surface. Bulletin of the South Ural State University. Series: Mathematical Modelling and Programming 7(2), 118-123 (2014) 